\title{
Le Traitement Médiatique du Transfèrement de Laurent Gbagbo à la Haye Par Trois Quotidiens Ivoiriens Entre 2011 et 2012
}

\author{
Dr. Celestin Gnonzion (Enseignant chercheur) \\ UFR, Information, Communication et Arts \\ Université Felix Houphouët Boigny d’Abidjan, Cocody, Côte d'Ivoire
}

doi: 10.19044/esj.2017.v13n11p18 URL:http://dx.doi.org/10.19044/esj.2017.v13n11p18

\begin{abstract}
This article aims to highlight the problems that are associated with a number of Ivoirian press. In the newsrooms, journalists basically handle information on the subject of politics with partiality. For instance, in the news coverage of the transfer of former President Laurent Gbagbo to The Hague, this paper shows how this fact was dealt with in three newspapers: 'Notre Voie', 'Le Nouveau Reveil', and 'Le Patriote'. Each of these newspapers treated the information according to the perception of leaders and politicians they want to satisfy. The article demonstrates that when the owner of the journal is influenced by politic power, it is difficult to have professionals in journalism who respect the journalist's code of ethic and deontology.
\end{abstract}

Keywords: Ivoirian press, international penal court, mistreatment of new information, Laurent Gbagbo, Côte d'Ivoire

\section{Résumé}

Cet article veut mettre en exergue le déséquilibre dans le traitement de l'information et la superficialité de certains quotidiens ivoiriens, surtout de ceux de notre échantillon: Notre Voie, le Nouveau Réveil, le Patriote. Dans le traitement de l'actualité portant sur le transfèrement de l'ancien président Laurent Gbagbo à la Haye, ces journaux se sont focalisés sur la justification ou la consolidation des opinions des mentors politiques dont ils sont proches. Ils avaient presque tous à cœur de défendre les positions d'hommes et de partis politiques au moment où les observateurs et lecteurs s'attendaient à une présentation et une explication des faits d'actualité portant sur la justice pénale internationale. Car le caractère nouveau, (du moins pour les ivoiriens) de problématiques soulevées par la justice 
transitionnelle et la justice pénale internationale méritaient d’être mieux expliqués aux citoyens et lecteurs.

Mots-clés : Presse ivoirienne, Cour Pénale Internationale, déséquilibre dans le traitement de l'information, Laurent Gbagbo

\section{Introduction}

\section{- $\quad$ Le Contexte}

Cet article s’inscrit dans le cadre de nos recherches sur la sociologie des médias et du journalisme en contexte ivoirien. Dans ces recherches, nous nous intéressons aux interactions entre les médias et les sources d’informations. Nous avons montré dans des travaux antérieurs (Gnonzion, 2008) que les sources d'informations, surtout, les sources politiques, tendaient à influencer les journalistes à travers des formes de corruption nommées par les journalismes eux-mêmes sous forme d'euphémismes comme «mesures d'accompagnement », «frais de transport » ou « perdiem » (Gnonzion, 2012).

C'est dans le prolongement des travaux sur les interactions entre les médias et les sources d'informations que nous abordons cet article en mettant cette fois-ci, l'accent sur les productions journalistiques ou le traitement de l'information par la presse dans un contexte politique donné. Il s'agit du contexte de la crise postélectorale de 2010.

Les élections présidentielles de 2010 en Côte d'Ivoire ont été marquées par une crise politico-militaire suite à laquelle, l'un des protagonistes, l'ancien président Laurent Gbagbo a été arrêté, transféré d'Abidjan, la capitale économique de la Côte d'Ivoire, à Korhogo, à près de $602 \mathrm{~km}$ au nord du pays, avant d'atterrir 7 mois semaines plus tard à la Haye pour y être jugé par la Cour Pénale Internationale. Le transfert de l'ancien président, d'une prison ivoirienne de Korhogo à celle de la CPI a été longuement couvert par les médias nationaux et internationaux. Et parmi ceux-ci, nous avons retenu la couverture médiatique de trois quotidiens ivoiriens : le Patriote, Le Nouveau Réveil et Notre Voie.

Cet article cherche à montrer comment ce fait d'actualité a été perçu et couvert médiatiquement par ces trois quotidiens aux lignes éditoriales proches d'idéologies de partis politiques en Côte d'Ivoire.

L’article présente dans un premier temps, le problème les objectifs de la recherche. L’approche théorique et le choix méthodologique sont ensuite exposés avant la présentation des résultats.

\section{- Le Problème}

Le problème que cet article veut mettre en exergue est celui du déséquilibre dans le traitement de l'information et quelques fois, la 
superficialité de certains quotidiens ivoiriens, surtout de ceux de notre échantillon. Dans le traitement de l'actualité portant sur le transfèrement de Laurent Gbagbo à la Haye, les journaux se sont focalisés sur la justification ou la consolidation des opinions des mentors politiques dont ils sont proches. Ils avaient presque tous à cœur de défendre les positions d'hommes et de partis politiques au moment où les observateurs et lecteurs s'attendaient à une présentation et une explication des faits d'actualité portant sur la justice pénale internationale. Car le caractère nouveau, (du moins pour les ivoiriens) de problématiques soulevés par la justice transitionnelle et la justice pénale internationale méritaient d’être mieux expliquées aux citoyens et lecteurs.

Pourtant et comme le souligne Blé Raoul Germain (2009), «Dans le contexte ivoirien, on peut donc, compte tenu du tribalisme et de l'ignorance (la population compte 65 pour cent d'analphabètes), faire l'hypothèse que les médias d'opinion peuvent exercer une influence davantage dans le sens du renforcement des opinions ».

Sur la base de cette hypothèse, il faut donc s’inquiéter lorsque des journaux qui, d'après cet auteur, renforcent les opinions politiques, ne le font pas dans le strict respect des normes professionnelles. Et Blé continue pour dire que certains journalistes ivoiriens pratiquent du journalisme « alimentaire qui se manifeste par la complaisance et la corruption ». Pour lui en effet, «bon nombre d'articles sont souvent des publi-reportages qui, comme " un griot », encensent les personnalités du pays ». Dans un tel contexte, ce sont les populations exposées à ces médias qui ne bénéficient pas d'informations approfondies pour participer de la meilleure manière possible au jeu démocratique, en faisant des choix politiques responsables.

Notons par ailleurs que le journaliste, peu importe la rédaction ou le journal dont il est collaborateur, qu'il s’agisse du journal d'un parti politique ou d'une personnalité politique, doit respecter les règles d'écriture journalistique Il doit par exemple séparer les faits des commentaires et ne pas confondre les genres journalistiques. Mais une fois avoir maîtrisé ces règles de base de l'écriture journalistique, le journaliste se doit également de connaitre et de respecter les codes d’éthique et de déontologie. En clair le journalisme est une profession à part entière. Et cette profession ne doit pas se confondre avec les autres professions comme nous l'apprenait le journaliste Dan Moussa, ancien président de l'Observatoire de la liberté de la presse, de l'éthique et de la déontologie, une instance d'autorégulation des médias en Côte d'Ivoire et ancien président de l’Union de la Presse Francophone.

«Le journaliste peut écrire sur les autres métiers dont la politique, mais il ne doit pas se substituer à ceux qui ont choisi de faire le métier de politique. Il ne doit pas se substituer aux politiciens. Quand il se substitue aux politiciens, il cesse d'être un journaliste, il n'est plus en train de faire du 
journalisme. Donc le fait qu'on veuille écrire sur le social, qu'on veuille écrire sur les assureurs, qu'on veuille écrire sur des banquiers, ne doit pas faire de nous, des cadres de banques, ne doit pas faire de nous des cadres d'assurances. Le fait qu'on écrive sur l'éducation ne doit pas faire de nous des enseignants. Le fait qu'on écrive sur les faits divers ne doit pas faire de nous des bandits ». (Gnonzion, $2008: 221$ )

Mais bien malheureusement, certains journalistes en Côte d'Ivoire, confondent leur métier avec celui des politiciens.

\section{- $\quad$ Les Objectifs de L'article}

Le premier objectif de cet article est de démontrer comment la presse ivoirienne est prisonnière des idéologies et des partis politiques. Et ceci, à travers la couverture d'un simple fait d'actualité. Le deuxième objectif est de montrer que depuis la crise politique et militaire de fin 2010 et début 2011 connue en Côte d'Ivoire, sous le nom de crise postélectorale, l'agenda des médias ivoiriens s'est enrichi d'un nouveau champ de traitement de l'actualité. Il s'agit des questions portant sur la justice internationale et le Tribunal Pénal International. Car suite à cette crise d'après élections, l'un des protagonistes à l'élection présidentielle de 2010, l'ancien président Laurent Gbagbo, a été transféré à la Cour Pénale Internationale.

Quelques années après, cet article se propose de revenir sur la perception de la justice internationale et sur le traitement émanant de l'actualité de cette juridiction internationale par quelques journaux ivoiriens.

L'approche choisie pour traiter ce problème est une approche qualitative basée sur une analyse documentaire et sur une analyse sémantique et lexicale du contenu des « Une » et des articles de trois quotidiens de notre échantillon.

La suite de l'article est organisée selon le plan suivant : une revue de la littérature, un cadre méthodologique et la présentation des résultats.

\section{L'approche Théorique}

Du point de vue théorique, nous avons abordé les prises de positions observées dans le traitement de l'actualité sur la CPI par ces trois quotidiens ivoiriens, sous l'angle du clientélisme politique et de l'interaction entre journalistes et hommes politiques en Côte d'Ivoire. Nous considérons les partis pris dans le traitement de l'actualité et le déséquilibre dans le traitement de l'information comme une forme de déviation professionnelle, ou de déviance tout court.

Emboîtant le pas à Becker (1985 : 16) et à Goffman (1975:12-13), deux auteurs de la troisième génération de l'Ecole de Chicago, nous pouvons dire que la déviance des productions de presse est un attribut des interactions entre les sources d'informations politiques et les journalistes. D'après cette 
perspective, et c'est l'une de nos hypothèses, il existerait une influence négative des hommes politiques sur les journalistes en Côte d'Ivoire. Cette influence est facilitée par le pouvoir que possèdent les hommes politiques et elle se manifeste sous forme de corruption, facilitée par le phénomène du clientélisme politique. Pour nous, le contexte et la réalité sociale des journalistes ivoiriens sont faits, certes de relations interpersonnelles avec les collègues journalistes, les chefs de service ou des journalistes de la vieille garde, mais surtout des relations avec les hommes politiques comme des sources d'information, mais aussi comme des bienfaiteurs, des propriétaires ou actionnaires des entreprises de presse.

C'est ici qu'il nous semble intéressant de souligner, à travers l'analyse des interactions entre les journalistes et les hommes politiques, le clientélisme qui semble être l'une des variables déterminantes qui conditionne la liberté des journalistes en Côte d'Ivoire. Le clientélisme, d'après ce qu'en dit LEGG (1975:4), met en liaison deux acteurs se trouvant à des situations sociales inégales. L'auteur nous apprend que « la relation de clientélisme est une relation de dépendance, établie et maintenue entre deux acteurs ayant des statuts inégaux, au niveau de la richesse, du pouvoir, du statut social.» Qu'en sera-t-il en Côte d'Ivoire, entre, d'une part, des journalistes se plaignant de leurs salaires jugés maigres, et, d'autre part, des hommes politiques détenteurs de certains pouvoirs et avantages sociaux et matériels que confèrent leurs fonctions politiques et administratives.

Ce clientélisme a été favorisé par le contexte du retour au multipartisme en 1990 qui offrait, du point de vue juridique, l'opportunité de la création des nouveaux journaux. Comme le souligne Ble Raoul (2006 :312) «L'Etat a permis la création des journaux sans pour autant prendre des dispositifs nécessaires pour les rendre suffisamment autonomes et responsables. Du coup, ces nouvelles et nombreuses rédactions sont récupérées par les pouvoirs politiques et les pouvoirs d'argent ». Et la presse ivoirienne peine toujours à se rendre responsable et professionnelle par l'appropriation de valeurs éthiques et à travers la pratique d'un journalisme soucieux du respect des codes de déontologie.

A la sociologie du contexte et des interactions (Becker, 1985) et (Goffman, 1975), à la question du clientélisme (Legg, 1975 : 4) et à celle du manque de l'autonomie des rédactions (Blé, 2006), on peut ajouter la théorie économique des médias (Flichy, 1980 : 240 ; Mosco, 1996 : 19, \& Chomsky, 1998). Pour les tenants de cette théorie sociologique d'analyse des médias, les relations entre la presse et les institutions politiques, sont un instant pendant lequel, les institutions des médias sont tenues par les institutions politiques grâce au pouvoir économique de ces derniers. Partant de ce postulat, les théoriciens économiques des médias pensent qu'il est difficile pour les hommes de la presse d'être indépendants, dans la mesure où ils ne 
sont pas libres économiquement. Pour quelques chercheurs de cette théorie comme Flichy, Mosco, ou Chomsky, les industries de la communication sont devenues des créateurs d'injustices entre les mains de multinationales aux visées d'exploitation et anti-démocratiques. Derrières les médias préexisteraient des logiques du business et des règles de l'autorité de l'Etat à travers l'économie capitaliste.

Chomsky et Edward Herman (1998) soutiennent par exemple, à travers leur «model de propaganda» que les médias comme des institutions de la démocratie ne peuvent pas bien jouer leurs trois principaux rôles, à savoir le rôle d'investigateur ou Watchdog, le rôle de combattant ou Adversary, et le rôle d'orientation de l'opinion ou Agenda Setter. Du point de vue de Noam Chomsky et Edward Herman, les médias ne pourront parfaitement jouer ces trois rôles énumérés plus haut, que s’ils sont libres et indépendants des pouvoirs politiques et économiques. Mais malheureusement, soutiennent-ils, les médias et la presse n’arrivent pas toujours à atteindre cet objectif dans les sociétés démocratiques modernes influencées par les lois du libre marché.

Dans la perspective théorique de Chomsky et Edward Herman, les médias modernes se montrent dans leurs pratiques quotidiennes, comme détournés de leur rôle souhaité de Watchdog-Adversary-Agenda Setter (BAGDIKIAN, 1983). D’après ces auteurs, les médias cherchent plutôt à créer un consensus, « manufacture consent » autour des idées et des idéologies de la classe dirigeante.

Après le cadre théorique, nous passons au traitement médiatique du transfèrement de Laurent Gbagbo à la Haye par les trois quotidiens de notre échantillon.

\section{Choix Méthodologique}

Avant d'aborder le cadre méthodologique, il importe de situer le contexte des productions journalistiques exploitées dans cet article. Il s’agit en effet d'un contexte social de bipolarisation de l'espace public. En Côte d'Ivoire, c’est comme si la presse a été piégée par les institutions politiques, de telle sorte qu'on note une polarisation des institutions de presse en deux grandes tendances ou camps politiques. Nous avons, d'un côté, une presse proche du parti au pouvoir et de l'autre, une presse qui se range derrière les partis d'oppositions. Mais il s'agira juste, dans une tentative de typologie selon les lignes éditoriales, de classer la presse ivoirienne en trois catégories: la presse proche des partis du Rassemblement des Houphouétistes pour la Démocratie et la Paix $\left(\mathrm{RHDP}^{37}\right)$, celle proche des

37 Le Rassemblement des houphouëtistes pour la démocratie et la paix (abrégé en RHDP) est une coalition de partis politiques ivoiriens, fondée le 18 mai $2005^{1}$. Cette coalition incarne principalement la droite ivoirienne d'obédience houphouétiste, c'est-à-dire 
partis du Front Populaire Ivoirien (FPI) de Laurent Gbagbo, et ensuite la presse dite indépendante ou équilibriste, pour emprunter un terme à la classification proposée par Aghi Bahi (2008). L’auteur faisait une typologie de la presse ivoirienne d'opinion et d'information, en journaux bleus, journaux proches du G7 et journaux équilibristes (qui n’appartiennent à aucun camp politique et se veulent indépendants). Dans la classification d'Aghi Bahi, les journaux bleus sont ceux proches du FPI et les journaux anciennement appelés du G7 sont aujourd’hui ceux du RHDP.

L’appellation G7 fait référence, d’après Aghi Bahi (2008), aux 7 groupements politico-militaires auxquels faisait face le FPI de Laurent Gbagbo, alors au pouvoir. De ces groupements, écrit l'auteur : "Quatre sont politiques et se regroupent sous l'appellation du Rassemblement des Houphouëtistes pour la Paix et la Démocratie: le Rassemblement Des Républicains (RDR), le Parti Démocratique de Côte d'Ivoire (PDCI), l'Union pour la Démocratie et la Paix en Côte d'Ivoire (UDPCI), et le Mouvement des Forces de l'Avenir (MFA). Trois sont militaires et se regroupent sous l'appellation Forces Nouvelles (FN). Il s'agit d'une coalition des ex-rebelles comprenant le Mouvement Patriotique de Côte d'Ivoire (MPCI), le Mouvement pour la Justice et la Paix (MJP) et le Mouvement Populaire Ivoirien du Grand Ouest (MPIGO). »

Après cette présentation du contexte politique de la presse en Côte d'Ivoire, nous pouvons aborder le cadre méthodologique de cet article.

L’univers de la presse écrite en Côte d'Ivoire, d'après les données du Conseil National de la Presse $^{38}$, est reparti entre 29 quotidiens, 24 hebdomadaires et 15 publications classés sous le vocable «autres périodicités ». De cet ensemble de journaux, nous avons choisi, sur la base d'un échantillon non probabiliste et par jugement, trois quotidiens d'informations générales et d'opinions : le Patriote, le Nouveau Réveil et Notre voie. Nous avons ensuite, à travers une approche qualitative, effectué une analyse thématique de contenu d'un corpus d'articles de ces trois quotidiens.

se réclamant de l'idéologie politique du fondateur de la Côte d'Ivoire, Félix HouphouëtBoigny. Le RHDP est une formation composée de cinq partis politiques : $\underline{\text { Parti démocratique }}$ de Côte d'Ivoire, dirigé par l'ancien président Henri Konan Bédié ; républicains, parti dont est issu l'actuel président Alassane Ouattara ;Union pour la démocratie en Côte d’Ivoire, dirigé par Albert Mabri Toikeusse ;Mouvement des forces d’avenir, dirigé par Moutayé Anzoumana. Union pour la Côte d'Ivoire, dirigé par Gnamien Konan.

(https://fr.wikipedia.org/wiki/Rassemblement_des_houphou\%C3\%ABtistes_pour_la_d\%C3 \%A9mocratie_et_la_paix)

38 Données recueillies sur le site du conseil national de presse, (http://www.lecnp.com/mag/?n=TGVzIEF1dHJlcyBw6XJpb2RpY2106XM=\&m=Mw==), visité le 28-01-2017. 
Il semble utile de préciser que les deux premiers quotidiens cités sont proches du point de vue idéologique et éditorial, successivement, du parti le Rassemblement des républicains (RDR) et du Parti démocratique de Côte d' Ivoire, Rassemblement Démocratique Africain (PDCI-RDA). Le troisième quotidien de notre échantillon, à savoir Notre Voie, est le journal du Front Populaire Ivoirien. Un tableau tiré d'une étude de Zio Moussa (2012), l'actuel président de l'Observatoire de la Liberté de la Presse, de l'Ethique et de la Déontologie (OLPED), nous permet de connaitre les propriétaires des journaux de notre échantillon.

\begin{tabular}{|c|c|c|c|}
\hline Titre/société éditrice & $\begin{array}{c}\text { Forme et } \\
\text { capital social }\end{array}$ & $\begin{array}{c}\text { Actionnaire } \\
\text { majoritaire/nombres de } \\
\text { parts }\end{array}$ & $\begin{array}{c}\text { Autres } \\
\text { actionnaire/nombres de } \\
\text { parts }\end{array}$ \\
\hline $\begin{array}{c}\text { Le Nouveau } \\
\text { Révil/Les éditions } \\
\text { le Reveil }\end{array}$ & $\begin{array}{c}\text { SARL au } \\
\text { capital de } \\
5000.000\end{array}$ & $\begin{array}{c}\text { Denis Kah Zion } \\
400 \text { parts }\end{array}$ & $\begin{array}{c}\text { Le personnel des éditions } \\
\text { le Réveil } \\
100 \text { parts }\end{array}$ \\
\hline $\begin{array}{c}\text { Notre Voie/ La } \\
\text { refondation }\end{array}$ & $\begin{array}{c}\text { SA au capital } \\
\text { de } \\
10.000 .000\end{array}$ & $\begin{array}{c}\text { Le FPI représenté par } \\
\text { Affi Nguessan } 800 \\
\text { parts }\end{array}$ & $\begin{array}{c}\text { Koffi Aka } 60 \text { parts ; } \\
\text { Odette Sauyet 60 parts ; } \\
\text { Allou Wanyou 40 parts ; } \\
\text { Danoh Djédjé 40 parts. }\end{array}$ \\
\hline $\begin{array}{c}\text { Le Patriote/Mayama } \\
\text { Edition production }\end{array}$ & $\begin{array}{c}\text { SARL au } \\
\text { capital de } \\
5000.000\end{array}$ & $\begin{array}{c}\text { Bakayoko Ahmed } \\
475 \text { parts }\end{array}$ & $\begin{array}{c}\text { Le personnel de Mayama } \\
\text { Edition production 25 } \\
\text { parts }\end{array}$ \\
\hline
\end{tabular}

Ce tableau indique clairement que des trois quotidiens de notre échantillon, seul le quotidien Notre Voie, a, comme propriétaire, un parti politique, le Front Populaire Ivoirien, représenté par un de ses cadres, en l'occurrence AFFI N'Guessan. Les deux autres quotidiens, le Nouveau Réveil et le Patriote ont comme propriétaires, des personnalités proches des partis politiques. Il s’agit de Bakayoko Ahmed pour le RDR et de Denis Kah Zion pour le PDCI-RDA.

Le matériau utilisé dans cet article est constitué de quelques publications de ces quotidiens après que l'ancien président Laurent Gbagbo soit transféré à la Haye. Nous avons sélectionné des publications au lendemain du transfèrement Laurent Gbagbo à la CPI, c'est-à-dire entre la période du 29 novembre 2011 au 12 décembre 2011. Nous avons également pris des publications durant l'année 2012 qui traitaient de la question de la justice transitionnelle et du tribunal pénal international en lien avec l'affaire Laurent Gbagbo. Ces publications ont été choisies sur la base d'un échantillonnage par objectif.

De ces publications des trois quotidiens sur les périodes indiquées, nous avons fait une analyse de contenu thématique et syntaxique, essentiellement, à partir des «UNE». Nous avons ensuite tenté une compréhension et une analyse des termes choisis par les journaux dans les 
éléments lexicaux et syntaxiques de leurs «UNES ». Il s’est agi donc d'une analyse soit lexicale, soit syntaxique de quelques éléments de notre corpus.

\section{Le traitement médiatique du transfèrement de Laurent Gbagbo à la CPI dans les quotidiens Le Patriote, le Nouveau Réveil, Notre Voie}

Dans le traitement de l'actualité portant sur les activités de la Cour Pénale Internationale, l'on a noté que la presse ivoirienne d'opinion est restée fidèle à sa réputation de presse partiale, superficielle, dont les prises de positions sont fortement orientées selon le bord idéologique de ses tuteurs politiques. La fin de la crise postélectorale a été marquée par la prise du pouvoir par le président Alassane Ouattara et l'arrestation, le 11 avril 2011 de l'ancien président Laurent Gbagbo, candidat malheureux aux élections présidentielles.

L’ancien président a ensuite été déporté à Korhogo, une ville du Nord de la Côte d'Ivoire avant d'être transféré à la Haye au Tribunal Pénal International, le mardi 29 novembre 2011, «en exécution du mandat d'arrêt $\mathrm{n}^{\circ}$ ICC-02/11 du 23 novembre 2011 et d'une demande d'arrestation et de remise $n^{\circ}$ ICC-02/11-01/11 en date du 25 novembre 2011 ». (Le Patriote du vendredi 02 décembre 2011).

La presse ivoirienne d'opinion a largement couvert l'actualité portant sur le transfèrement de Laurent Gbagbo, mais également, celle de ces différentes auditions devant la Cour Pénale Internationale. Nous avons, pour les besoins de notre analyse, retenu de nous intéresser à quelques « Une » de Le Patriote, de Le Nouveau Réveil et de Notre Voie.

\section{Le patriote}

Dans son traitement de l'actualité liée au transfèrement de Laurent Gbagbo à la CPI, le quotidien proche du RDR d'Alassane Ouattara, a tenté d'orienter son analyse sur le bienfondé de cette décision mais surtout sur le fait que Laurent Gbagbo n'est pas indispensable à la Côte d'Ivoire, car la vie continue sans lui : "Gbagbo à la Haye, son parti est sans chef: FPI BORIBANA. La tête du serpent est coupée la vie continue en Côte d'Ivoire ». (Le Patriote du 02 décembre 2011). On peut observer à partir de ce titre du Patriote, un certain nombre d’éléments :

Premièrement, le quotidien fait remarquer à ses lecteurs que le FPI n'a plus désormais de chef. Cet accent mis par le journal sur l'absence du leader du FPI est évocateur d'une sorte de satisfaction du journal, dans la mesure où il compare ce parti politique à un "serpent » dont la « tête est coupée ».

Deuxièmement, le Patriote se sert du transfèrement de Laurent Gbagbo à la Haye, pour adresser un message symbolique, non seulement à ses lecteurs supposés, mais également à toute la Côte d'Ivoire, à travers le sous-titre «FPI BORIBANA », qui veut dire «FPI la course est terminée » 
en langue bambara (« dioula ») . Le bambara est une langue ivoirienne parlée par une bonne partie des populations du Nord de la Côte d'Ivoire. Il est aussi la langue véhiculaire ivoirienne, qui sert de moyen de communication à plusieurs populations analphabètes parlant diverses langues ivoiriennes. Le choix de l'usage d'une expression dans cette langue véhiculaire de la Côte d'Ivoire, ne peut viser qu'un public de lecteurs pas forcément alphabétisés à la lecture du message médiatique.

Le Patriote continue son analyse de l'actualité de la CPI portant sur Laurent Gbagbo en accusant ce dernier de mentir aux juges de cette institution: "Première comparution de Gbagbo devant la CPI .Tout est faux !voici le mensonge ». (Le Patriote du 6 décembre 2011).

Le quotidien oriente son traitement de la première comparution de Laurent Gbagbo devant la CPI en se situant du côté de l'accusation. Il écrit en s'exclamant que "tout est faux! ».Une manière d'attirer l'attention de l'accusation qui doit redoubler d'efforts face à un prévenu proférant du « mensonge ». Si ce quotidien ne tente pas d'intervenir à la Haye en secours à l'accusation, c'est sur le terrain en Côte d'Ivoire même, qu'il continue son accusation à l'endroit Laurent Gbagbo qu'il qualifie de «tyran » et dont le bilan est publié par voie de presse : « 3000 morts et 2 millions de déplacés ». (Le Patriote du 29 décembre 2011)

Laurent Gbagbo est dépeint dans le Patriote comme un sanguinaire, le " tueur des femmes aux mains nues ». (Le Patriote du 3 mars 2012). Pour tous ces crimes, le quotidien proche du RDR réclame justice en se posant contre la libération de l'ancien président pourtant exigée par le parti de ce dernier : " En exigeant la libération de Gbagbo et les ex dirigeants, le FPI se moque des 3000 morts de la crise ». (Le Patriote du 12 et 13 mai 2012). Le FPI demande en effet que tous les prisonniers issus de son rang y compris Laurent Gbagbo soient libérés. La raison qui fonde cette exigence est le contexte de réconciliation et de reconstruction post-crise.

Mais Le Patriote se montre à travers ses écrits et sa ligne éditoriale opposée à la libération des prisonniers de la crise postélectorale. Il publie dans son édition du jeudi 02 février que «les pro-Gbagbo jugés avant décembre » et ajoute que «La justice sera rendue au nom du peuple de Côte d'ivoire et de façon équitable, nul n'est au-dessus de la loi ». (Le Patriote du 2 février 2012).

On peut retenir en conclusion que le quotidien Le Patriote, dans le traitement de l'actualité concernant les activités de la CPI, adopte une position éditoriale en faveur de la justice internationale. Car pour ce quotidien, les personnes soupçonnées par la justice (nationale ou internationale) doivent tous répondre des accusions portées contre elles. 


\section{Le nouveau reveil}

Le nouveau Réveil, proche du PDCI, l'allié du RDR a un traitement de l'actualité portant sur les activités de la CPI similaire à celui de Le Patriote. Dès les premiers jours du transfèrement de Gbagbo la Haye, le quotidien publie en prenant position que « La CPI est mieux pour Gbagbo ». (Le Nouveau Réveil du 02 décembre 2011). Il emboite également le pas à Le Patriote dans l'accusation de Laurent Gbagbo, avec une manière propre au quotidien proche d’Henri Konan Bédié, d’enfoncer davantage l’accusé, en enrichissant les arguments de l'accusation : «Première comparution devant la CPI [...] «Gbagbo, première spirale de mensonge, le tueur de 3000 ivoiriens se fait passer pour une victime ». (Le Nouveau Réveil du 06 décembre 2011).

Le quotidien utilise des termes et expressions hostiles à l'accusé Laurent Gbagbo, auteur de «spirale de mensonge » et «tueur de 3000 ivoiriens ». Un tel accusé mérite de séjourner à la CPI. Et le journal de prédire que Gbagbo ne sortira pas de la prison de la Haye : « Gbagbo sortir de la Haye ? Le FPI rêve debout ». (Le Nouveau Réveil du 20 mai 2012). On voit donc que dans la mise en scène de la réalité, chaque quotidien prend l'angle d'analyse selon l'idéologie de son tuteur politique, mais aussi pour écrire selon l'attente de ses lecteurs militants.

Le Patriote et le Nouveau Réveil, deux quotidiens proches du RHDP, par le choix des mots de leurs UNES respectives, ont cherché:

- $\quad$ À légitimer l'arrestation de l'ancien président Laurent Gbagbo.

- $\quad$ À tenter de démontrer sa culpabilité.

- $\quad$ À montrer qu'il ne sera pas libérer.

Et ils le font à travers un choix d'expressions et de mots très hostiles à l'ancien président et en l'accusant directement.

\section{Notre voie}

Nous avons noté au niveau de le Patriote et du Nouveau Réveil que la tendance éditoriale est en faveur de la justice, nationale comme internationale. Pour ces deux quotidiens proches du RHDP, toute personne soupçonnée d’être impliqué en tant qu'auteur dans les crimes liés à la crise postélectorale doit répondre de ces actes devant les juridictions. C'est au nom de cet attachement à la justice que les deux quotidiens ont salué le transfèrement de Laurent Gbagbo devant la CPI et écrit des articles pour soutenir l'accusation en enfonçant le prévenu.

Le quotidien Notre Voie, proche du FPI, le parti fondé par Laurent Gbagbo, tente plutôt de démontrer que l'ancien président est non seulement détenu de façon illégale à la Haye, mais attire l'attention sur le fait que la CPI est partiale car instrumentalisée. Dans sa parution du 2 décembre 2011, presqu'au lendemain du transfèrement de Laurent Gbagbo à la CPI, le 
quotidien pointait déjà du doigt sur l’idée d’une justice de vainqueur, car les crimes du camp Ouattara restent impunis : "Soro avoue le complot: Le procureur Simplice KOFFI nie les crimes du camp Ouattara ». (Notre Voie du 2 décembre 2011)

Mais Notre Voie tente de jouer sur la psychologie des masses populaires en faveur de l'ancien président lorsqu'il titre : « Gbagbo aux Ivoiriens ne pleurez pas soyez forts » (Notre Voie du 1 décembre 2011). Le quotidien met en scène et présente Gbagbo comme une victime, mais une victime très forte psychologiquement, car non seulement elle supporte et assume l’épreuve d’un transfèrement "illégal », mais elle se soucie de la masse des ivoiriens, qu'elle cherche d'ailleurs à réconforter " soyez forts ». En clair, dans sa couverture de l'actualité des activités de la CPI concernant le procès de Laurent Gbagbo, Notre Voie présente ce dernier comme un héros, non seulement national, mais un héros sur la scène internationale et africain : « Gbagbo livré à la CPI L’Afrique divisée » (Notre Voie des 13 et 14 décembre 2011). Il est présenté comme le père de la démocratie ivoirienne, comme un homme politique qui a toujours prôné le dialogue : " Première comparution à la Haye Gbagbo va parler à la CPI aujourd'hui. [...] Promoteur de la démocratie, il a toujours prôné la voie pacifique à travers le slogan «asseyons-nous et discutons » (Notre Voie du 15 décembre 2011).

L’accusé Laurent Gbagbo dépeint par les quotidiens proches du RHDP comme le tueur qui mérite de demeurer en prison à la Haye est présenté par Notre Voie, comme une victime injustement incarcérée. Il est décrit comme un démocrate, comme celui qui a toujours préconisé la voie du dialogue, un pacifique auteur du slogan « « asseyons-nous et discutons ».

Le journal s’en prend par ailleurs au président de la Commission Dialogue, Vérité Réconciliation (CDVR) accusé de ne rien faire pour empêcher la «justice à deux vitesses ». Face à l'appel du président de la CDVR qui demande à ce que «tous les Ivoiriens, ainsi que, toutes les communautés vivant sur le sol de Côte d'Ivoire quelle que soit leur sensibilité, à regarder ensemble dans la même direction ». Notre Voie répond en se demandant:

« Mais comment tous les Ivoiriens pourraient-ils regarder dans la même direction quand les vainqueurs d'une guerre se croient autorisés à faire ce qu'ils veulent des vaincus et que même le réconciliateur en chef n'a pas le courage de dénoncer la justice à deux vitesses ? " (Notre Voie du 09 décembre 2011).

Notre Voie fera de la dénonciation de cette « justice à deux vitesses », une stratégie de sa communication en faveur de la libération de Laurent Gbagbo, ou pour la déportation à la CPI des personnalités proches du camp adverse. 
C’est pourquoi le journal donnera un large écho aux différents rapports des ONG internationales de défense des droits de l'Homme: Amnesty international et Human Rights Watch, qui dénonçaient justement une justice des vainqueurs en Côte d'Ivoire. Dans la titraille de Notre Voie du $1^{\text {er }}$ Mars 2012, on pouvait lire en effet : « Crimes massifs des hommes de Ouattara/ Le rapport explosif qui dévoile tout / Voici pourquoi Ouattara et Soro n'échapperont pas à la CPI » (Notre Voie du 01 Mars 2012).

En conclusion, on peut retenir que le quotidien Notre Voie, proche du FPI, dans son traitement de l'actualité concernant le transfert et le jugement de Laurent Gbagbo à la Haye, a tenté d'informer et de communiquer sur trois points:

- L L'illégalité du transfèrement de Laurent Gbagbo à la Haye

- $\quad$ La présentation de Laurent Gbagbo comme une victime innocente aux mains d'une institution manipulée

- $\quad$ L'application d'une justice à deux vitesses, car seul un camp est visé pendant que des personnalités du camp adverse impliquées dans la crise postélectorale sont en liberté.

\section{Conclusion}

Cet article visait deux objectifs majeurs. Le premier était de démontrer, à travers la couverture d'un fait d'actualité, que certains journaux ivoiriens, comme ceux de notre échantillon sont prisonniers des idéologies de partis politiques dont ils sont proches, au détriment d'un traitement plus professionnel et objectif de l'information. Le second objectif était de montrer que l'agenda de la presse ivoirienne s'est enrichi de nouvelles thématiques portant sur la justice pénale internationale, notamment à travers le transfert et le jugement de Laurent Gbagbo à la Cour pénale internationale.

Nous avons tenté de montrer, sur la base d'une interprétation de quelques UNES, que dans leur couverture de l'actualité portant sur les activités de la CPI, les trois quotidiens que sont Le Patriote, Le Nouveau Réveil et Notre Voie, ont limité leur traitement aux aspects idéologiques et partisans. Ils ont fait le choix de ne pas aborder le débat de fond sur la justice transitionnelle et la justice tout court, qu'elle émane des juridictions nationales ou interactionnelles. Les journaux ont simplement cherché a enfoncé ou a encensé une autorité politique selon sa proximité idéologique avec le journal.

C'est d'ailleurs cette superficialité dans le traitement de l'information de certains journaux que Germain Ble Raoul (2006) dénonçait lorsqu’il écrivait que «les journalistes véhiculent des informations fantaisistes qui en font des "poisons humains" de conditionnement social ».

Ces traitements partisans des faits d'actualité confirment également ce que disait Nyamnjoh (2000) qui notait que dans certains pays comme le 
Sénégal, le Mali, le Niger, la Côte d’Ivoire et le Rwanda, des journaux ont contribué à l'incitation à la division et à la haine. L'auteur fera en outre remarquer qu'entre 1990 et 1993, les media furent hautement politisés au Cameroun et polarisés entre deux camps diamétralement opposés. Chaque camp réclamant connaître et représenter au mieux les intérêts de la société et du peuple. " Pour chaque camp, le Dieu, les anges et les saints de l'autre camp ne sont rien d'autre que Lucifer et démons».

Or pour un pays comme la Côte d'Ivoire, qui sortait d'une crise postélectorale de la violence de celle de 2010, la presse et les médias devraient jouer leur rôle de promotion de la démocratie, de la réconciliation, pour la consolidation de la paix.

\section{References:}

1. Aghi Bahi Auguste \& Lori-Anne Theroux-Benoni (2008). « À propos du rôle des médias dans le conflit ivoirien ", HTTP://WWW.CERIUM.CA/IMG/PDF/2008_THEROUX-BENONI_BAHIA_PROPOS_DU_ROLE_DE_SMEDIA10-COTEDIVOIRE-2.PDF (VISITE LE 17 OCTOBRE 2013)

2. Bagdikian, B. H. (1983). The Media Monopoly, Boston: Beacon Press.

3. Blé Raoul Germain (2006). «Médias d'opinions et crise ivoirienne ", Les Enjeux de l'information et de la communication, 1/2006 (Volume 2006), p. 3-12.

4. -------------------- «La guerre dans les médias, les médias dans la guerre en Côte d'Ivoire », Afrique et développement, Vol. XXXIV, No. 2, 2009, pp. 177-201.

5. Becker, H.S. (1985). Outsiders : études de sociologie de la déviance, Paris : Metaillié, 1985, p.16

6. Edward, H. \& Chomsky,N. (1998). «Manufactured consent » in O'NEIL, P. H., Communicating democracy : the media and political transitions, London: Rienner.

7. Flichy, P. (1980). Les industries de l'imaginaire : pour une analyse économique des media, Grenoble : Presses Universitaires de Grenoble, p. 240.

8. Gnonzion Celestin (2008). Les facteurs de décisions éthiques et de construction de l'identité des journalistes ivoiriens, Thèse de doctorat, Université Grégorienne de Rome, 2008.

9. ----------------«Perdiems, gombos et mesures d'accompagnement: les mots et les rites de la corruption dans la presse ivoirienne », European Scientific Journal (ESJ), vol 8, $\mathrm{N}^{\circ} 23$ (2012).

10. Goffman, E. (1975). Stigmate, les usages sociaux des handicaps, Paris, Minuit, pp. 12-13. 
11. Legg, R., K. (1975). Patrons, clients and politicians, Bekerley (CA): University of California. Institute of international studies.

12. Le Patriote du vendredi 02 décembre 2011, «Transfèrement de Laurent Gbagbo à la CPI / Simplice Kouadio Koffi (Procureur de la République : "Chacun paie pour ce qu'il a fait” » in

13. le Patriote du 29 décembre 2011, «Côte d’Ivoire: Bilan d'une apocalypse avortée »,

14. le Patriote du 3 mars 2012 , «Il y a 1 an, Gbagbo tuait des femmes aux mains nues ",

15. Le Patriote du 12 et 13 mai 2012, «En exigeant la libération de Gbagbo et les ex dirigeants, Le FPI se moque des 3000 morts de la crise »

16. Le Patriote du 2 février 2012, «Crise postélectorale: Les proGbagbo jugés avant décembre »,

17. Le Nouveau Réveil du 02 décembre 2011 « La CPI est mieux pour Gbagbo ».

18. Le Nouveau Réveil du 06 décembre 2011, « Première comparution devant la CPI »,

19. Le Nouveau Réveil du 20 mai 2012, « Gbagbo sortir de la Haye ? Le FPI rêve debout »

20. Mosco, V. (1996). The political economiy of communication: rethinking and renewal, London: Sage Publications.

21. Notre Voie du 2 décembre 2011, «Soro avoue le complot: Le procureur Simplice KOFFI nie les crimes du camp Ouattara ».

22. Notre Voie du 1 décembre 2011, « Gbagbo aux Ivoiriens ne pleurez pas soyez forts ",.

23. Notre Voie des 13 et 14 décembre 2011, «Gbagbo livré à la CPI L’Afrique divisée ",

24. Notre Voie du 15 décembre 2011, « Première comparution à la Haye Gbagbo va parler à la CPI aujourd'hui ».

25. Notre Voie du 09 décembre 2011, «Banny tente de banaliser la déportation de Gbagbo

26. Notre Voie du 01 Mars 2012, « Crimes massifs des hommes de Ouattara/ Le rapport explosif qui dévoile tout / Voici pourquoi Ouattara et Soro n'échapperont pas à la CPI ».

27. Nyamnhoh, F.B. (2000). «West Africa: Unprofessional and unethical journalism», In MICHAEL KUNCZIK (ed.), Ethics in journalism: a reader on their perception in the Third World, The Division for International Development Cooperation of Friedrich-Ebert-Stiftung, Bonn: FES Library. 
28. Zio Moussa (2012). Les Médias et la Crise Politique en Côte d'Ivoire, FONDATION POUR LES MÉDIAS EN AFRIQUE DE L'OUEST, Legon, Ghana. 